\title{
A narrative review of gallbladder adenomyomatosis: what we need to know
}

\author{
Kit-Fai Lee ${ }^{1}$, Esther H. Y. Hung ${ }^{2}$, Howard H. W. Leung ${ }^{3}$, Paul B. S. Lai ${ }^{1}$ \\ ${ }^{1}$ Division of Hepatobiliary and Pancreatic Surgery, Department of Surgery, Prince of Wales Hospital, the Chinese University of Hong Kong, Hong \\ Kong, China; ${ }^{2}$ Department of Imaging and Interventional Radiology, Prince of Wales Hospital, the Chinese University of Hong Kong, Hong Kong, \\ China; ${ }^{3}$ Department of Anatomical and Cellular Pathology, Prince of Wales Hospital, the Chinese University of Hong Kong, Hong Kong, China \\ Contributions: (I) Conception and design: KF Lee, PBS Lai; (II) Administrative support: KF Lee; (III) Provision of study materials or patients: All \\ authors; (IV) Collection and assembly of data: All authors; (V) Data analysis and interpretation: KF Lee; (VI) Manuscript writing: All authors; (VII) \\ Final approval of manuscript: All authors. \\ Correspondence to: Dr. Kit-Fai Lee. Department of Surgery, Prince of Wales Hospital, the Chinese University of Hong Kong, 30-32, Ngan Shing \\ Street, Shatin, N.T., Hong Kong, China. Email: leekf@surgery.cuhk.edu.hk.
}

\begin{abstract}
Gallbladder adenomyomatosis (GA) is increasingly encountered in clinical practice due to increasing use of imagings especially ultrasound (US). Clinicians need to know what is the implication of this condition and its proper management. GA is a degenerative and proliferative disease characterized by excessive epithelial proliferation associated with hypertrophy of muscularis propria. This leads to outpouchings of mucosa into or beyond the muscle layer forming intramural diverticula recognized as Rokitansky-Aschoff sinuses (RAS). Three types of GA are recognised: fundal, segmental and diffuse type. In fundal GA, there is focal thickening involving the GB fundus. In segmental GA, there is circumferential overgrowth of the GB wall that leads to formation of compartments. In diffuse GA, there is disseminated thickening and irregularity of the mucosa and muscularis. The pathogenesis of GA is unknown. It commonly occurs in middle age with equal sex distribution. Diagnosis of GA is by imagings showing thickened gallbladder wall containing cysts. Characteristic features are "comet-tail" artefacts and "twinkling" artefacts on US, "pearl-necklace sign" on magnetic resonance imaging (MRI) and "rosary sign" on computed tomography (CT). Cholecystectomy should be offered for symptomatic GA of any type. For asymptomatic GA, cholecystectomy may be considered for segmental type for its increased risk of malignancy and for diffuse type for its difficult visualization of any coexisting malignancy. Asymptomatic fundal GA can be safely observed with US. How frequent and how long should a fundal GA be monitored with US remains unknown. In case of diagnostic doubt, cholecystectomy should always be offered to avoid overlooked malignancy.
\end{abstract}

Keywords: Gallbladder adenomyomatosis (GA); gallbladder carcinoma; gallbladder lesion; gallbladder wall thickening

Submitted Jun 23, 2020. Accepted for publication Sep 25, 2020.

doi: 10.21037/atm-20-4897

View this article at: http://dx.doi.org/10.21037/atm-20-4897

\section{Introduction}

Abdominal ultrasound (US) is a commonly ordered investigation in surgical practice. Not uncommonly there is incidental finding of gallbladder (GB) wall thickening containing cysts on US, a condition called gallbladder adenomyomatosis (GA). Though GA is increasingly encountered, most clinicians know little about the disease and its proper management.

The key questions are: is GA alone an indication for surgery? Is GA a premalignant lesion? How should GA be followed up and with what type of imaging? Is long term 


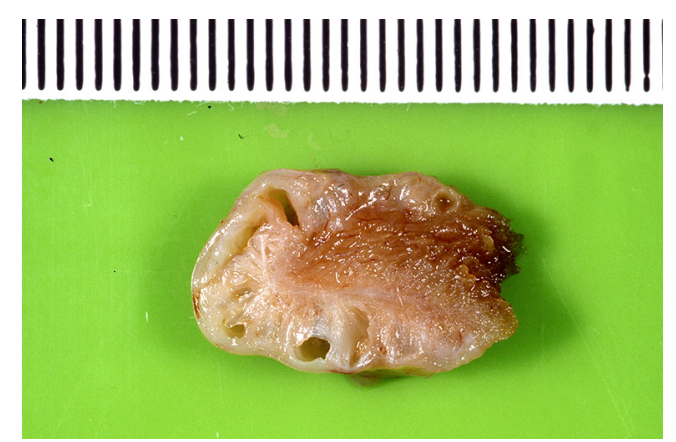

Figure 1 Trimmed gross specimen of fundal type gallbladder adenomyomatosis reveals thickened gallbladder wall with multiple intramuscular cystic spaces.

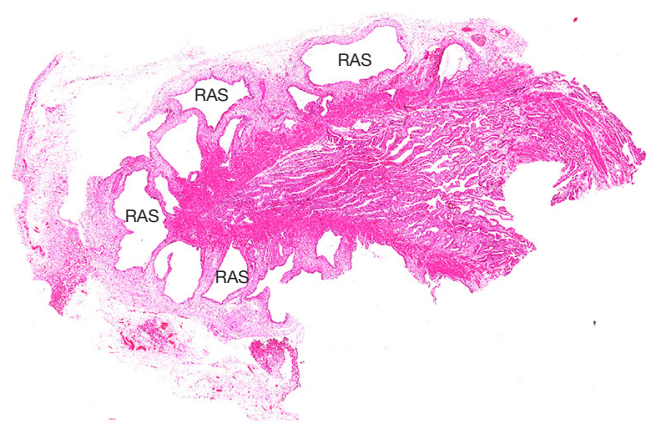

Figure 2 The corresponding histology shows cystically dilated glands invaginating into the thickened muscular wall forming the Rokitansky-Aschoff sinuses (RAS). Hematoxylin and eosin; magnification $20 \times$.

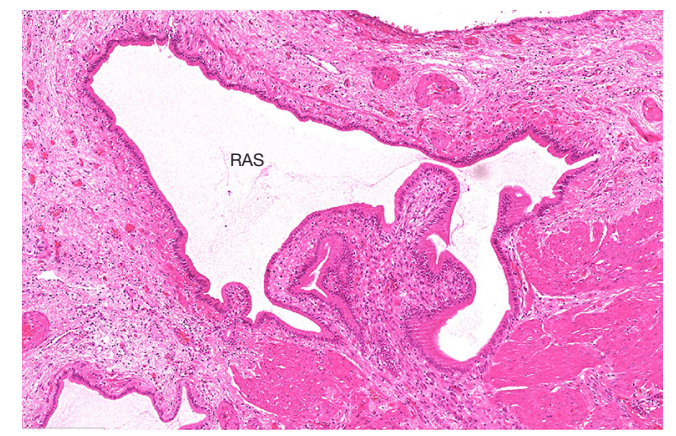

Figure 3 The cystically dilated glands consist of the same benign epithelial lining as normal biliary mucosa. RAS, RokitanskyAschoff sinuses. Hematoxylin and eosin; magnification 100×. follow-up necessary for GA?

To answer these questions, an extended non-systemic literature search of PubMed articles in English with the search item of GA was performed. Original articles, reviews and cases reports on GA up to April 2020 were included. A narrative synthesis of extracted data was performed and presented using basic thematic analysis. We present the following article in accordance with the Narrative Review reporting checklist (available at: http://dx.doi.org/10.21037/ atm-20-4897).

\section{Overview}

GA is a pathological condition characterized by excessive epithelial proliferation associated with hypertrophy of muscularis propria, resulting in GB wall thickening (1-3). The excessive epithelial proliferation leads to outpouchings of mucosa into or beyond the muscle layer forming intramural diverticula (cysts on US), recognized as Rokitansky-Aschoff sinuses (RAS) which was first described by Rokitansky in 1842 and then by Aschoff in 1905 (Figures 1-3) (4,5). RAS may contain calculi or cholesterol crystals. The serosa is never involved by GA (6).

Different names have been used for GA which included hyperplasic adenomyosis, adenomyoma, adenofibromyoma, cystic cholecystitis, proliferative glandular cholecystitis, intramural diverticulosis, hamartoma and diverticular disease of GB, until 1960 Jutras used the term GA to define this condition which was a degenerative and proliferative disease (7).

Depending on the degree of GB wall involvement, GA is classified into three types: fundal, segmental or diffuse (Figure 4). In fundal GA, there is focal thickening involving the GB fundus while the rest of GB appears physiologically thin and GB shape is maintained. In segmental GA, there is circumferential overgrowth of the GB wall that leads to formation of compartments within GB, resembling an "hourglass" appearance. In diffuse GA, there is disseminated thickening and irregularity of the mucosa and muscularis, resulting in cyst-like appearance of GB $(6,8)$. Sometimes, a segmental type GA with its segment near the gallbladder neck can be confused with the diffuse type. A further complex type may also be included when there is a coexistence of a 


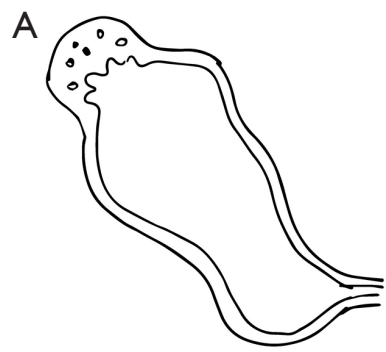

B

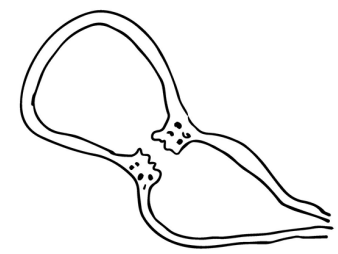

C

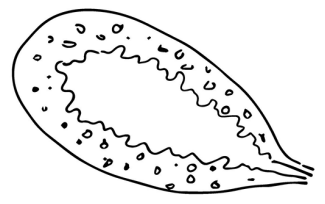

Figure 4 Schematic drawing of different types of gallbladder adenomyomatosis: (A) fundal; (B) segmental; (C) diffuse.

segmental and fundal type GA. A previous study showed that the segmental type is the commonest while in one recent study, the fundal type is the commonest GA $(9,10)$.

\section{Epidemiology}

GA is typically diagnosed beyond the age of 50-60, with similar sex distribution or female predominance $(2,11)$. It was found in $2 \%$ to $8 \%$ of all cholecystectomies in recent series and in $1-5 \%$ in autopsy $(10,12,13)$.

\section{Pathogenesis}

The pathogenesis of GA is unknown. It is unlikely to be a congenital malformation as fewer than 10 cases have been reported in children in first year of life (14-16). GA is widely accepted to be a degenerative disease (17). Increased intra-luminal pressure secondary to neurogenic dysfunction may play a role $(1,18)$. Anomalous pancreaticobiliary ductal union which causes chronic reflux of pancreatic fluid into GB may explain the occurrence of GA $(19,20)$. Coexisting gallstone (GS) occur in more than $50 \%$ of patients with GA and in up to $90 \%$ of segmental GA but no causative relationship has been proved $(9,12)$. In latter case, GS are located predominantly in fundal compartment in around $80 \%$ of cases, which is likely secondary to biliary stasis (9).

\section{Diagnosis}

Majority of GA cases are incidentally discovered by imaging or by pathology of resected GB. Patients with GA are usually asymptomatic. The most common symptom is nonspecific abdominal pain localised at the right upper quadrant and epigastrium. The pain is similar to that of gallstone and is typically intermittent and self-limiting (21). Nausea, vomiting, fatty food intolerance can also occur.
However, symptoms can also be attributed to the commonly coexisting GS. GA presented as acalculous cholecystitis has been reported (22). Laboratory tests in patients with GA are usually normal.

\section{US}

GB wall thickening $(>3 \mathrm{~mm})$ represents a hallmark of GA, being always present but is poorly specific $(6,23)$. The outer GB layer must appear sharp and a clear cleavage plane with liver must be present (6). Small anechoic cystic spaces (1-10 mm) representing clear bile-filled RAS within GB wall are pathognomonic for GA. When RAS are filled with cholesterol crystals, intramural echogenic foci are noted (6). These cholesterol crystals, in contrast to the clear bile within the RAS, act as highly reflective surfaces and generate posterior reverberation artefacts on grey-scale US-the so-called "comet-tail" artefacts. It is characterized by a bright hyperechoic focus at the GB wall with an inverted triangular acoustic enhancement posteriorly, which exhibit a gradual decrease in strength and thickness (Figure 5) (23). Comet-tail artefact is a reverberation artefact on grey scale US which occurs when two closely packed echogenic interface are struck by US beam. This is a typical finding of GA as the aggregates within the RAS act as the echogenic interface, but the phenomenon is not limited to GA. Other examples with aggregate serving as reflective interface within small cystic lesions producing this artefact include milk of calcium cyst in the kidneys and biliary hamartoma in the liver. Resembling the "comettail" artefact on grey scale US, another artefact known as the "twinkling" artefact which appears as a rapid alternation of blue and red Doppler signals immediately deep to the highly reflective cholesterol crystal is seen on color Doppler US (24). Limitation of US lies on its operator dependence and imaging artefact due to presence of gas or stones and 

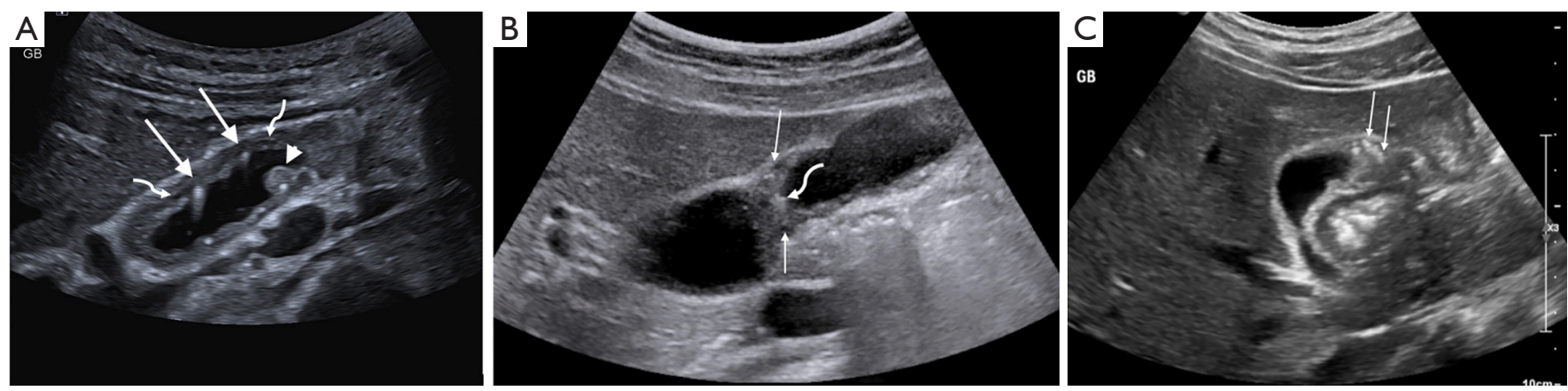

Figure 5 The ultrasound appearance of different types of gallbladder adenomyomatosis. (A) Grey scale ultrasound image showing a diffusetype gallbladder adenomyomatosis with diffuse wall thickening containing multiple small cysts (curved arrows) representing RAS and comettail artefacts (arrows) representing cholesterol aggregates. Note the small polypoid lesion (arrow head) representing focal mural hypertrophy associating with adenomyomatosis. (B) Grey scale ultrasound imaging showing a segmental type of gallbladder adenomyomatosis with focal annular wall thickening at the mid body, giving rise an hourglass appearance of the gallbladder. Note the small RAS (arrows) and comet tail artefacts (curved arrow). (C) Grey scale ultrasound imaging showing a fundal type of gallbladder adenomyomatosis with localized involvement at the gallbladder fundal wall and multiple comet-tail artefacts (arrows).

inadequate $\mathrm{GB}$ visualization in obese patients.

High resolution US $(2.5-7 \mathrm{MHz})$ and contrast US using micro-bubble contrast material have been reported to increase the diagnostic accuracy for differentiation of GA and gallbladder cancer (GC) (6,25). Endoscopic US (EUS) is credited with better images of GB than usual US. EUS has been reported to identify GA that are missed by transabdominal US $(26,27)$. Nevertheless, EUS may misdiagnose GC as GA due to microcysts that can also be seen in mucin producing GC $(28,29)$.

\section{Computed tomography $(C T)$}

A confident diagnosis of GA in thickened GB wall is possible only if large (at least $3 \mathrm{~mm}$ ) RAS are present. A diagnostic sign of GA on CT is the "rosary sign", which occurs from combination of an unenhanced proliferative muscularis layer surrounding enhanced proliferative mucosal epithelium with intramural diverticula (Figure 6) (30). Another sign called "cotton ball sign" may also present. This is seen as fuzzy grey enhancing dots in a thickened GB wall or a dotted outer border of the inner enhancing layer of GB wall on contrast CT and is more sensitive when the RAS are small (31).

\section{Magnetic resonance imaging (MRI)}

In GA, GB wall thickening can be clearly depicted both on T1- and T2-weighted images. RAS typically appears markedly hyperintense on T2-weighted images, hypointense on T1-weighted images and show no contrast enhancement. A distinguished sign of GA on T2-wighted image is the "pearl-necklace sign", which is the presence of multiple high intensity cavities seen in GB wall (Figure 7) $(6,30)$. This sign is more prominent on magnetic resonance cholangiopancreatography (MRCP) $(6,32)$.

\section{Positron emission tomography (PET)}

GA typically shows no 18 -fluorodeoxyglucose $\left({ }^{18} \mathrm{FDG}\right)$ uptake (SUV <2.5) or lower uptake compared with liver while GC is usually hypermetabolic. However, acute inflammatory reaction surrounds RAS may generate an increased ${ }^{18} \mathrm{FDG}$ uptake and give rise to false positive result. PET is not performed for diagnosis of GA, but may help to exclude GC when there is no significant tracer uptake at the lesion (33).

\section{Choice of imaging}

US should be the imaging modality of choice in initial evaluation for GA. MRI represents the ideal tool for further evaluation in case of inconclusive finding on US. CT can be used as a diagnostic adjunct to evaluate GB wall thickening and to differentiate GA and GC. The accuracy to differentiate GA from early GC for US, MRI and CT were $91-95 \%, 93 \%$ and $40-75 \%$ respectively (6). EUS, being invasive and costly, should be used selectively. For PET, 


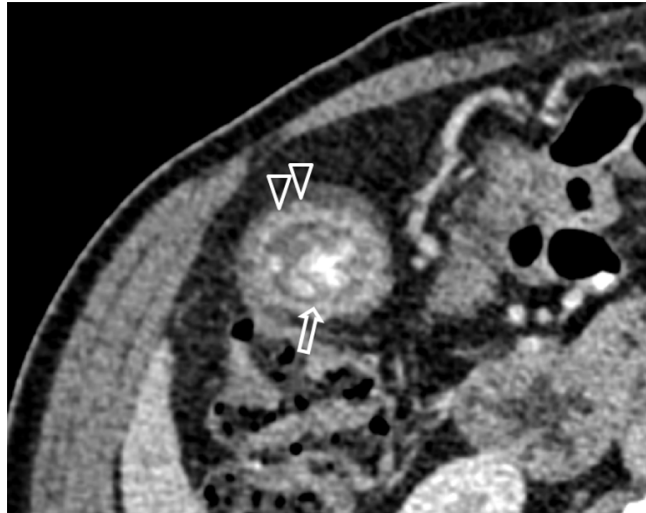

Figure 6 Computed tomography image shows the "rosary sign". The hyper-enhanced inner lining (arrow) represents the proliferative mucosa. Note the multiple mucosa-lined cystic outpouching (arrowheads) invaginating into the hypertrophied muscularis propria representing the RAS. Hyperdense gallstones are present. RAS, Rokitansky-Aschoff sinuses.

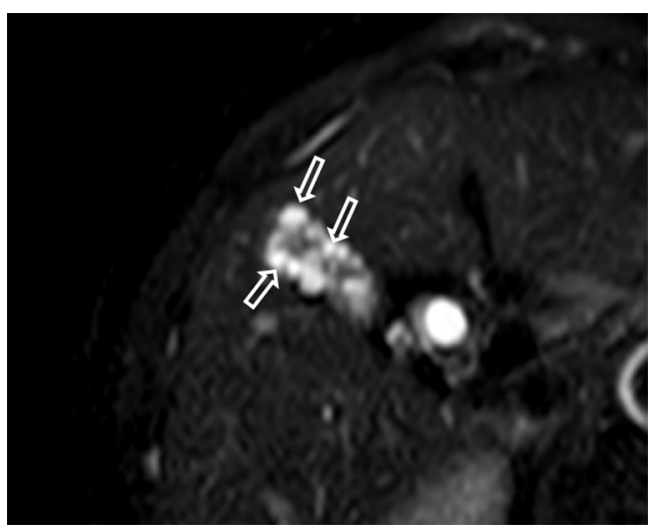

Figure 7 Magnetic resonance imaging shows multiple hyperintense, fluid-filled intramural diverticula (arrows) in the gallbladder fundus representing the "pearl-necklace sign".

there is no evidence for its routine use. For differential diagnosis of GA, an MRI or contrast CT is necessary, whilst for follow-up for GA, US is usually sufficient.

\section{Association with GC}

GA has all along been considered as a benign condition. However, cases of coexisting GC and GA have been reported in the literature (34-36). GC arose both from just above fundal GA and the distal part of segmental GA (37). Ootani et al. reported that GC developed in 6.4\% (12/188) of patients with segmental GA, whereas no clear association was found between fundal and diffuse type GA with GC (12). The tumor in patients with both segmental GA and GC only occurs in the fundic mucosa rather than the neck mucosa. It was postulated that the segmental GA led to bile congestion and intra-luminal hypertension in the distal GB leading to chronic inflammation which was regarded as a cancer causing factor. Nabatame et al. also found that $6.6 \%(22 / 334)$ of patients with segmental GA had GC (38). An association between dysplastic adenoma and diffuse GA has also been described (39). Other authors suggested GA could not be regarded as precancerous lesion based on available evidence, but stones and cholecystitis secondary to GA might lead to dysplastic change and cancer (40).

\section{Management}

There is no universally accepted guideline for the management of GA at present. The questions are how reliable is the diagnosis of GA on imaging and how high is the chance of malignant change for a genuine GA.

GA only accounts for $25 \%$ of cases of GB wall thickening ( $>3 \mathrm{~mm}$ ), important differential diagnosis of GC needs to be excluded (8). US being frequently the initial imaging has its own limitations. When characteristic imaging findings are not present, it may be challenging to distinguish GA from GC. MRI is the preferred next imaging of choice if US is inconclusive. The radiological diagnosis of GA must be beyond any doubt, otherwise cholecystectomy with intraoperative frozen section should be offered to exclude GC. Extended surgery should be planned in advance if GC is confirmed intra-operatively.

For symptomatic GA with or without concomitant cholecystolithiasis, cholecystectomy is indicated as all reported cases were effectively and durably relieved by cholecystectomy (2).

In view of increased risk of GC, cholecystectomy may be considered for segmental type of GA even without symptom $(12,38)$ and for diffuse type as it carries the problem of difficult visualization of coexisting malignancy (39). For fundal type GA, whatever its size and concomitant gallstone, surgery is not indicated as no series reported an increase risk of GC in cases of exclusively fundal GA (8). Other authors recommended fundal GA to be dealt with in the same way as for GB polypoid lesions, i.e., for cholecystectomy if lesion $\geq 10 \mathrm{~mm}$ (17). However, it is arguable to equivalent GA to polypoid lesion of GB 


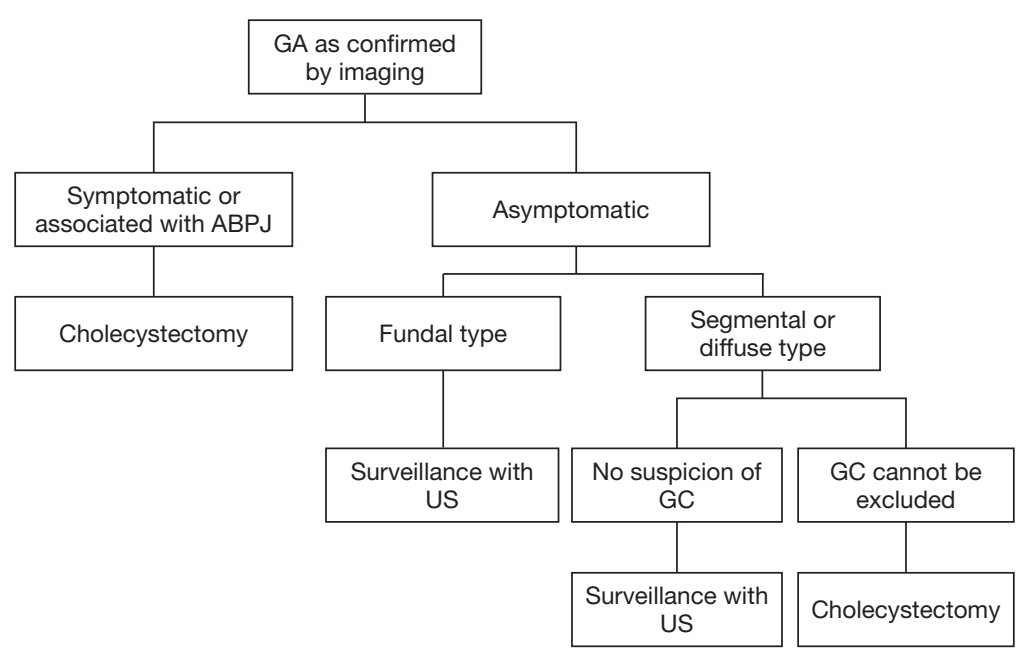

Figure 8 A proposed algorithm for the management of gallbladder adenomyomatosis. GA, gallbladder adenomyomatosis; GC, gallbladder cancer; US, ultrasound; ABPJ, anomalous biliopancreatic junction.

as the latter consisted of a very heterogenous group of histologically different diseases (41). Finally, the presence of concomitant anomalous biliopancreatic junction (ABPJ) with GA also presents an indication for cholecystectomy as ABPJ is a known risk factor for GC $(42,43)$. A proposed algorithm for the management of GA is shown on Figure 8.

Whether there is need for regular follow-up US for asymptomatic fundal GA and how frequent US should be done is unknown. A 3-6 monthly US follow-up has been suggested by some authors, but how long should this surveillance be carried on is again unknown $(17,44)$. Patient compliance for need of long-term surveillance is a problem. Resources implication also becomes a problem as GA is increasingly detected on US examination. Finally, GA may also increase in size over time and this change by itself cannot be considered an index of malignancy (45).

\section{Conclusions}

GA is increasingly encountered in clinical practice. A background knowledge of the disease and its management cannot be over-emphasized. Appropriate selection of patients for further investigation and surgery is crucial to avoid overlooked malignant disease and to treat potential malignant type GA whilst unnecessary surgery can be avoided in most benign types GA. Updated evidence shows that in general fundal and diffuse type GA are not premalignant whilst segmental type carries risk of GC. But for any type of GA, presence of symptom or associated ABPJ is an indication for cholecystectomy. Surgery should also be considered whenever GC cannot be excluded. US is the choice of imaging for follow up of GA with an interval of 3-6 months but how long should this surveillance be carried on remains to be answered.

\section{Acknowledgments}

Funding: None.

\section{Footnote}

Reporting Checklist: The authors have completed the Narrative Review reporting checklist. Available at: http:// dx.doi.org/10.21037/atm-20-4897

Peer Review File: Available at: http://dx.doi.org/10.21037/ atm-20-4897

Conflicts of Interest: All authors have completed the ICMJE uniform disclosure form (available at: http://dx.doi. org/10.21037/atm-20-4897). The authors have no conflicts of interest to declare.

Ethical Statement: The authors are accountable for all aspects of the work in ensuring that questions related to the accuracy or integrity of any part of the work are appropriately investigated and resolved. 
Open Access Statement: This is an Open Access article distributed in accordance with the Creative Commons Attribution-NonCommercial-NoDerivs 4.0 International License (CC BY-NC-ND 4.0), which permits the noncommercial replication and distribution of the article with the strict proviso that no changes or edits are made and the original work is properly cited (including links to both the formal publication through the relevant DOI and the license). See: https://creativecommons.org/licenses/by-nc-nd/4.0/.

\section{References}

1. Colquhoun J. Adenomyomatosis of the gall-bladder (intramural diverticulosis). Br J Radiol 1961;34:101-12.

2. Meguid MM, Aun F, Bradford ML. Adenomyomatosis of the gallbladder. Am J Surg 1984;147:260-2.

3. Williams I, Slavin G, Cox A, et al. Diverticular disease (adenomyomatosis) of the gallbladder: a radiologicalpathological survey. Br J Radiol 1986;59:29-34.

4. von Rokitansky C. Handbuch der speziellen pathologischen Anatomie. Vol II. Vienna: Braumiller \& Seidal; 1842:1-374.

5. Aschoff L. Bemerkungen zur pathologischen Anatomie des cholelithiasis und Cholecystitis. Verh Dtsch Pathol Ges 1905;9:41.

6. Bonatti M, Vezzali N, Lombardo F, et al. Gallbladder adenomyomatosis: imaging findings, tricks and pitfalls. Insights Imaging 2017;8:243-53.

7. Jutras JA. Hyperplastic cholecystoses; Hickey lecture, 1960. Am J Roentgenol Radium Ther Nucl Med 1960;83:795-827.

8. Golse N, Lewin M, Rode A, et al. Gallbladder adenomyomatosis: Diagnosis and management. J Visc Surg 2017;154:345-53.

9. Nishimura A, Shirai Y, Hatakeyama K. Segmental adenomyomatosis of the gallbladder predisposes to cholecystolithiasis. J Hepatobiliary Pancreat Surg 2004;11:342-7.

10. Kim JH, Jeong IH, Han JH, et al. Clinical/pathological analysis of gallbladder adenomyomatosis; type and pathogenesis. Hepatogastroenterology 2010;57:420-5.

11. Joshi JK, Kirk L. Adenomyomatosis. StatPearls [Internet]. Treasure Island (FL): StatPearls Publishing; 2020-2019; Dec 30

12. Ootani T, Shirai Y, Tsukada K, et al. Relationship between gallbladder carcinoma and the segmental type of adenomyomatosis of the gallbladder. Cancer 1992;69:2647-52.
13. Arbab AA, Brasfield R. Benign tumors of the gallbladder. Surgery 1967;61:535-40.

14. Cetinkursun S, Surer I, Deveci S, et al. Adenomyomatosis of the gallbladder in a child. Dig Dis Sci 2003;48:733-6.

15. Zarate YA, Bosanko KA, Jarasvaraparn C, et al. Description of the first case of adenomyomatosis of the gallbladder in an infant. Case Rep Pediatr 2014;2014:248369.

16. Parolini F, Indolfi G, Magne MG, et al. Adenomyomatosis of the gallbladder in childhood: A systematic review of the literature and an additional case report. World J Clin Pediatr 2016;5:223-7.

17. Pellino G, Sciaudone G, Candilio G, et al. Stepwise approach and surgery for gallbladder adenomyomatosis: a mini-review. Hepatobiliary Pancreat Dis Int 2013;12:136-42.

18. Beilby JO. Diverticulosis of the gall bladder. The fundal adenoma. Br J Exp Pathol 1967;48:455-61.

19. Tanno S, Obara T, Maguchi H, et al. Association between anomalous pancreaticobiliary ductal union and adenomyomatosis of the gall-bladder. J Gastroenterol Hepatol 1998;13:175-80.

20. Chang LY, Wang HP, Wu MS, et al. Anomalous pancreaticobiliary ductal union--an etiologic association of gallbladder cancer and adenomyomatosis. Hepatogastroenterology 1998;45:2016-9.

21. Ram MD, Midha D. Adenomyomatosis of the gallbladder. Surgery 1975;78:224-9.

22. Lin SH, Chang FY, Yang YS, et al. Rare gallbladder adenomyomatosis presenting as atypical cholecystitis: case report. BMC Gastroenterol 2011;11:106.

23. Oh SH, Han HY, Kim HJ. Comet tail artifact on ultrasonography: is it a reliable finding of benign gallbladder diseases? Ultrasonography 2019;38:221-30.

24. Hammad AY, Miura JT, Turaga KK, et al. A literature review of radiological findings to guide the diagnosis of gallbladder adenomyomatosis. HPB (Oxford) 2016;18:129-35.

25. Joo I, Lee JY, Kim JH, et al. Differentiation of adenomyomatosis of the gallbladder from early-stage, wallthickening-type gallbladder cancer using high-resolution ultrasound. Eur Radiol 2013;23:730-8.

26. Azuma T, Yoshikawa T, Araida T, et al. Differential diagnosis of polypoid lesions of the gallbladder by endoscopic ultrasonography. Am J Surg 2001;181:65-70.

27. Sugiyama M, Atomi Y, Yamato T. Endoscopic ultrasonography for differential diagnosis of polypoid gall bladder lesions: analysis in surgical and follow up series. Gut 2000;46:250-4. 
28. Kim HJ, Park JH, Park DI, et al. Clinical usefulness of endoscopic ultrasonography in the differential diagnosis of gallbladder wall thickening. Dig Dis Sci 2012;57:508-15.

29. Yoshimitsu K, Irie H, Aibe H, et al. Well-differentiated adenocarcinoma of the gallbladder with intratumoral cystic components due to abundant mucin production: a mimicker of adenomyomatosis. Eur Radiol 2005;15:229-33.

30. Bang SH, Lee JY, Woo H, et al. Differentiating between adenomyomatosis and gallbladder cancer: revisiting a comparative study of high-resolution ultrasound, multidetector CT, and MR imaging. Korean J Radiol 2014;15:226-34.

31. Yang HK, Lee JM, Yu MH, et al. CT diagnosis of gallbladder adenomyomatosis: importance of enhancing mucosal epithelium, the "cotton ball sign". Eur Radiol 2018;28:3573-82.

32. Haradome H, Ichikawa $T$, Sou H, et al. The pearl necklace sign: an imaging sign of adenomyomatosis of the gallbladder at MR cholangiopancreatography. Radiology 2003;227:80-8.

33. Suzuki K, Watada S, Yoko M, et al. Successful diagnosis of gallbladder carcinoma coexisting with adenomyomatosis by 18 F-FDG-PET--report of a case. J Gastrointest Cancer 2011;42:252-6.

34. Aldridge MC, Gruffaz F, Castaing D, et al. Adenomyomatosis of the gallbladder. A premalignant lesion? Surgery 1991;109:107-10.

35. Funabiki T, Matsumoto S, Tsukada N, et al. A patient with early gallbladder cancer derived from a Rokitanski-Aschoff sinus. Surg Today 1993;23:350-5.

36. Suzuki K, Abe K, Ohbu M. A resected gallbladder carcinoma coexisting with adenomyomatosis involving varied degrees of intraepithelial dysplasia: a case report and literature review. Surg Laparosc Endosc Percutan Tech

Cite this article as: Lee KF, Hung EHY, Leung HHW, Lai PBS. A narrative review of gallbladder adenomyomatosis: what we need to know. Ann Transl Med 2020;8(23):1600. doi: 10.21037/atm-20-4897
2019;29:290-6.

37. Okaniwa S. Role of conventional ultrasonography in the diagnosis of gallbladder polypoid lesions. J Med Ultrason (2001) 2019. doi: 10.1007/s10396-019-00989-5

38. Nabatame N, Shirai Y, Nishimura A, et al. High risk of gallbladder carcinoma in elderly patients with segmental adenomyomatosis of the gallbladder. J Exp Clin Cancer Res 2004;23:593-8.

39. Di Carlo I, Toro A, Pulvirenti E, et al. An unusual association of diffuse adenomyomatosis with dysplastic adenoma in chronic calculous cholecystitis: case presentation. BMC Gastroenterol 2010;10:41.

40. Pang L, Zhang Y, Wang Y, et al. Pathogenesis of gallbladder adenomyomatosis and its relationship with early-stage gallbladder carcinoma: an overview. Braz J Med Biol Res 2018;51:e7411.

41. Lee KF, Wong J, Li JC, et al. Polypoid lesions of the gallbladder. Am J Surg 2004;188:186-90.

42. Chang J, Jang JY, Kang MJ, et al. Clinicopathologic differences in patients with gallbladder cancer according to the presence of anomalous biliopancreatic junction. World J Surg 2016;40:1211-7.

43. Funabiki T, Matsubara T, Miyakawa S, et al. Pancreaticobiliary maljunction and carcinogenesis to biliary and pancreatic malignancy. Langenbecks Arch Surg 2009;394:159-69.

44. Pang L, Wu S, Kong J. Surgical Choice for Different Types of Gallbladder Adenomyomatosis: An Initial Experience of 20 Years Laparoscopic Cholecystectomy. Surg Laparosc Endosc Percutan Tech 2020;30:151-5.

45. Agrawal S, Khurana J, Daruwala C. Gallbladder adenomyomatosis: a malignant masquerader. Dig Liver Dis $2012 ; 44: \mathrm{e} 23$. 